\title{
Natural Coatings on Titanium Surfaces to Improve Their Biological Response
}

\author{
Sara Ferraris 1, Enrica Verné 1, Gissur Örlygsson 2, Paulo Tambasco ${ }^{3}$, Felipe Perraro Sehn ${ }^{3}$, \\ Chuen-How Ng ${ }^{4}$, Hilmar Janusson ${ }^{4}$, Giuliana Banche ${ }^{5}$, Valeria Allizond ${ }^{5}$, \\ Cinzia Margherita Bertea ${ }^{5}$, Lia Rimondini ${ }^{6}$, Andrea Cochis ${ }^{6}$, Vincenzo Guarino 7 , \\ Alessio Varesano ${ }^{8}$, Claudia Vineis ${ }^{8}$, Giovanna Gautier di Confiengo ${ }^{9}$ and Silvia Spriano ${ }^{1}$ \\ 1 Politecnico di Torino-ITALY; \\ 2 Innovation Center Iceland - ICELAND; \\ 3 University of Sao Paulo - BRAZIL; \\ 4 Genis hf. - ICELAND; \\ 5 Università di Torino - Italy; \\ Università del Piemonte Orientale - Italy; \\ CNR-IPCB, Naples - Italy; \\ CNR-STIIMA Biella - Italy, \\ 9 CNR-IMAMOTER - Italy \\ * Correspondence: sara.ferraris@polito.it
}

Received: 28 February 2020; Accepted: 13 May 2020; Published: 14 May 2020

\begin{abstract}
Biomolecules and extracts from natural products are gaining increasing interest due to their beneficial properties for human health, low toxicity, environmental compatibility and sustainability.

In this work, keratin, chitosan and peppermint essential oil have been used for the preparation of coatings on titanium substrates for biomedical implants/devices. All these coatings were obtained from local natural products/byproducts: keratin from discarded wool, chitosan from shrimp shells and peppermint essential oils from a local production. The above cited molecules were selected for their ability to stimulate soft tissue adhesion (keratin), anti-inflammatory activity (chitosan) and antibacterial activity (keratin after metal ion doping, chitosan and mint oil).

The coatings were characterized by means of SEM-EDS, FTIR, zeta potential, wettability, tape and scratch tests, cell and bacteria cultures.

The coatings were successfully obtained for all the considered natural substances with good adhesion to the titanium substrates. All the coatings are chemically stable in water and the continuous coatings are mechanically resistant and protective for the metallic substrates. The keratin coatings are hydrophilic while the mint oil and chitosan coatings are hydrophobic; nanofibers instead of continuous coatings behave as more hydrophobic. At the physiological $\mathrm{pH}$, the keratin and mint oil coatings are negatively charged, when in contact with an aqueous environment, while the chitosan ones are positively charged. The oriented keratin fibers are able to drive fibroblast alignment. The Ag-doped keratin fibers and mint coating show antibacterial properties.
\end{abstract}

Keywords: titanium; keratin; chitosan; peppermint essential oil; coating

\section{Introduction}

Natural products are gaining increasing interest in the field of medicine due to their numerous beneficial properties (such as antioxidant, antibacterial, anti-inflammatory) and few side effects (e.g. limited toxicity and bacterial resistance). Moreover, they can be often obtained from abundant byproducts of different industries (food and beverages or textile to cite some examples). 
Among natural products, keratin, chitosan and peppermint essential oils have been selected in the present research as possible substances for the obtainment of coatings of interest in the biomedical field.

Keratin has been selected for its ability to stimulate soft tissue growth $[1,2]$ and the possibility to be doped with metal ions to confer antibacterial activity [3]. Chitosan has been selected for its antibacterial and antioxidant properties [4] and peppermint essential oil for its antibacterial properties [5].

All these substances were obtained from industrial byproducts or local products in order to promote a sustainable use of resources, with the transformation of byproducts in high added value products and to sustain local economies.

In this view, keratin was obtained from discarded wool, chitosan from shrimp shells and peppermint essential oil from the local mint production.

Keratin, chitosan and peppermint essential oil were used to prepare organic natural coatings onto commercially pure titanium and Ti6Al4V alloy. Samples were characterized by means of SEMEDS, FTIR, zeta potential, wettability, tape and scratch tests, cell and bacteria cultures.

\section{Materials and Methods}

Keratin was obtained by discarded wool from the textile industry by sulfitolysis with sodium metabisulfite, purified and freeze-dried in powder, as described in [6]. Keratin coatings, in the form of random or oriented sub-micrometric fibers, on polished or grooved commercially pure titanium surfaces, were obtained, from keratin solutions in formic acid, by electrospinning as described in [6,7]. Moreover, keratin continuous coatings were obtained on polished commercially pure titanium substrates, from keratin solution in water, by manual application and spreading of a drop of solution and further drying in air. All the coatings were thermally treated $2 \mathrm{~h}$ at $180^{\circ} \mathrm{C}$ to confer them stability [8]. Part of the random oriented submicrometric fiber coatings were soaked in silver nitrate aqueous solution in order to obtain silver doping [3].

Chitosan was obtained from shrimps' shells (Genis hf). Continuous chitosan coatings were prepared onto chemically treated Ti6Al4V substrates. The chemical treatments [9] foresees an acid etching followed by a controlled oxidation to obtain a nanotextured surface rich in hydroxyl groups. Chitosan grafting was performed directly, using tresyl chloride as good outgoing group or using polydopamine as linker.

Peppermint essential oil coatings were performed on chemically treated Ti6Al4V substrates (as for chitosan coatings) by means of application and spreading of a drop of pure oil and further drying at $37^{\circ} \mathrm{C}$ in an incubator [10].

Surface morphology and semi-quantitative chemical composition were investigated by means of Scanning Electron Microscopy equipped with Energy Dispersive Spectroscopy (FESEM-EDS SUPRATM 40, Zeiss and Merlin Gemini Zeiss). The appearance and distribution of mint essential oil coating (which is autofluorescent) was investigated by means of fluorescent microscopy (Zeiss LSM900, Zeiss, Oberkochen, Germany). The characteristic chemical groups of the coating molecules were further determined by means of Fourier Transformed Infrared Spectroscopy (Tensor 37 microFTIR with Hyperion 2000 Microscope-Bruker Optics, Ettlingen, Germany). The adhesion of the coatings to the substrates was evaluated by means of tape test (ASTM D 3359-97) and scratch tests (CSM, Revetest machine). The wettability of the coatings was evaluated by means of contact angle measurements (sessile drop method, DSA-100, KRÜSS GmbH, Hamburg, Germany) with ultrapure water as wetting fluid.

Surface charge in function of $\mathrm{pH}$ was determined by zeta potential electrokinetic measurements (SurPASS, Anton Paar, adjustable gap cell) in $0.001 \mathrm{M} \mathrm{KCl}$ titrated with $0.05 \mathrm{M} \mathrm{HCl}$ and $0.05 \mathrm{M} \mathrm{NaOH}$.

The antibacterial activity of silver doped keratin coatings and of peppermint essential oil coatings was evaluated against Staphylococcus aureus, since it is one of the most widespread pathogens $[3,10]$. 
The biocompatibility and fibroblast guidance ability of submicrometric fiber keratin coatings was evaluated with human gingival fibroblasts, because the application of these materials is intended in contact with soft tissues $[3,6,7]$.

\section{Results and Discussion}

The appearance of the various coatings is shown in Figure 1 through SEM micrographs for keratin and chitosan coatings (obtained by direct grafting) and fluorescence microscopy image for peppermint essential oil, as examples.

The SEM micrographs of the continuous keratin (Figure 1a) and chitosan coatings (Figure 1c) and the fluorescence image of the peppermint essential oil coating (Figure 1d) highlight homogeneous and substantially smooth coatings (except of sub micrometric spot like features in the keratin coating). On the other hand, as expected, the keratin electrospun coatings are characterized by sub-micrometric mainly regular fibers. It can be noticed that all the continuous coatings completely cover the substrate topography, exposing to the biological environment topography and chemistry of the coatings. Differently, the sub-micrometric keratin fibers can be modulated in order to completely cover the substrate or partially expose it; this can be of interest if the substrate features are a complementary stimulus for the cells, as previously demonstrated by the authors with aligned submicrometric keratin fibers deposited on grooved titanium substrates [7].
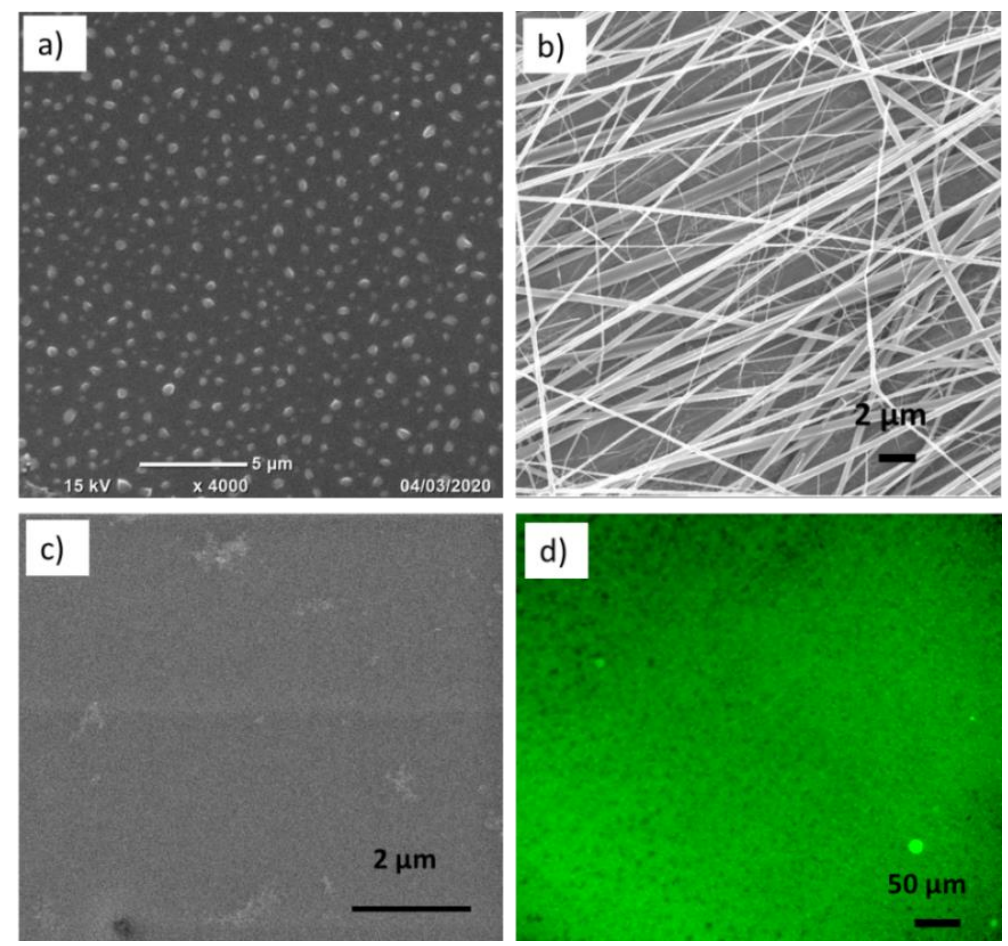

Figure 1. SEM micrographs of (a) keratin continuous coating, (b) keratin electrospun sub-micrometric fibers coating, (c) chitosan (direct grafting) continuous coating and (d) fluorescence microscopy image of peppermint essential oil coating.

The FTIR spectra (not reported) confirm for all the tested samples the effectiveness of the coating, evidencing the presence of the characteristic vibration peaks of the keratin, chitosan and peppermint essential oil constituents on the coated surfaces. All the coatings resulted stable in water and FTIR spectra (as well as SEM and fluorescence observations) confirm their presence after two weeks soaking in aqueous media.

The adhesion of the continuous keratin coatings, chitosan and peppermint essential oil coatings to the titanium substrates is optimal (5B classification according to ASTM D 3359-97 standard, no detachment). A certain reduction of adhesion (compared to the continuous coatings) has been evidenced for the fibrous keratin coating, and it can be associated to the lower availability of contact 
points for adhesion. Proper surface treatments are currently under investigation to take also these coatings to the same standard of the continuous ones.

The scratch tests evidenced a good adhesion for the continuous keratin coatings and peppermint essential oil coatings, confirming the tape test results. Moreover, these tests evidenced a significant protection ability of the coating for the metallic substrate. In fact, despite of the plastic deformation of the metal during the test (due to the application of a progressive load up to $10 \mathrm{~N}$ with a diamond indenter), no damages of the surface occurred on the coated samples, differently from the uncoated ones [11].

Water wettability of the here investigated coatings is reported in Figure 2.

The keratin continuous coatings are more hydrophilic than the titanium substrate. This behavior can be associated with the hydrophilic nature of keratin, reported in the literature [12]. On the other hand, the sub-micrometric fiber keratin coatings show an increase in the contact angle which can be attributed to the peculiar topography, rather than to the coating chemistry [6]. Both the chitosan and peppermint essential oil coatings are more hydrophobic than the Ti6Al4V substrate, and this behavior can be mainly associated to their chemistry. The contact angle measurements resulted an effective simple technique to characterize the water wettability of these materials and to confirm and verify the coating presence.

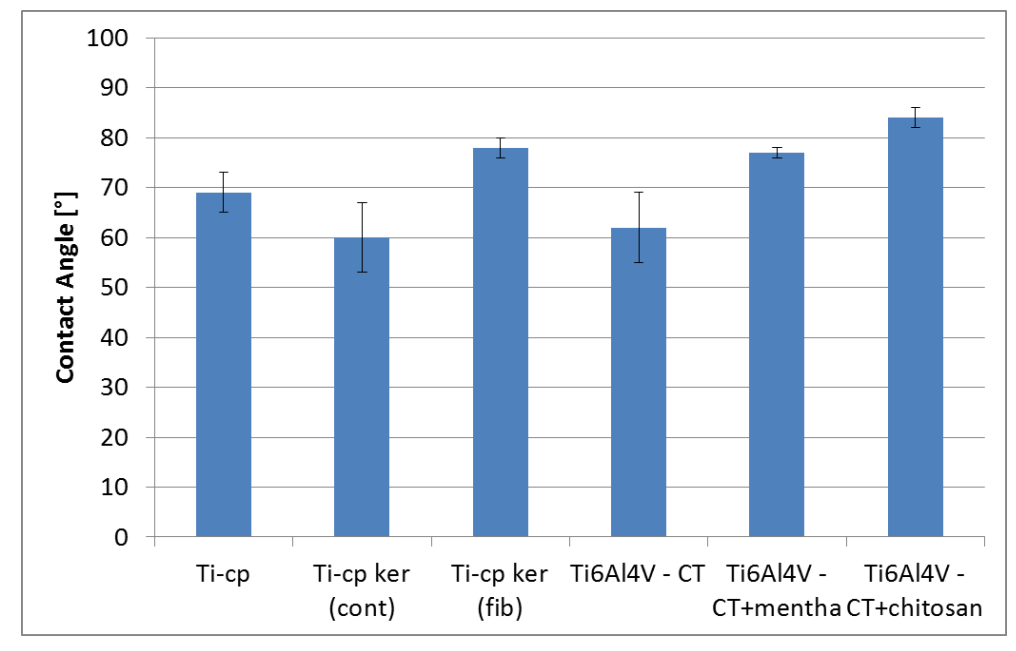

Figure 2. Water wettability of the different tested coatings.

Maintenance of surface hydrophobicity confirmed permanence of the chitosan and peppermint essential oil coatings on the titanium substrates after soaking in water based media.

The zeta potential titration curves of the bare and coated substrates are reported in Figure 3.

Figure $3 \mathrm{a}$ shows the zeta potential vs $\mathrm{pH}$ of commercially pure titanium bare or keratin coated. Ti-cp has an isoelectric point close to 4 (as reported in the literature [13]) consistent with the absence of specific functional groups. The introduction of keratin, both as continuous coating or as submicrometric fibers, shifts the isoelectric point to more acidic values ( 3.4 and 2.8 respectively) and exposes functional groups with acidic behavior ( $\mathrm{COOH}$ of the keratin molecules). The presence of these acidic functional groups is confirmed by the plateau in the basic region, observable in both curves.

The chemically treated Ti6Al4 alloy (Ti6Al4V - CT) has a very acidic IEP (close to 2 and obtained only by curve interpolation) due to exposition of the acidic $\mathrm{OH}$ groups, as previously reported by the authors $[14,15]$. The mint essential oil coating shift the IEP close to 4 in accordance with the absence of specific charged functional groups. This feature is confirmed by the absence of a plateau in the curve. Finally, chitosan coating shifts the IEP to basic values (8.5) in accordance with the IEP of chitosan [16] and the presence of basic amino- groups. In this case, only the measurement of zeta potential in the basic range was possible due to chitosan swelling at moderately acid $\mathrm{pH}$. 
The bare substrates, keratin and mint essential oil coatings have acidic IEPs and consequently are negatively charged at the physiological $\mathrm{pH}$. On the other hand, chitosan has a basic IEP and consequently it is the only surface positively charged at the physiological $\mathrm{pH}$.

All the measurements, except of keratin continuous coating at $\mathrm{pH}$ 3.7, show a very small standard deviation, which can be associated with their chemical stability in aqueous media in a wide $\mathrm{pH}$ range [14].
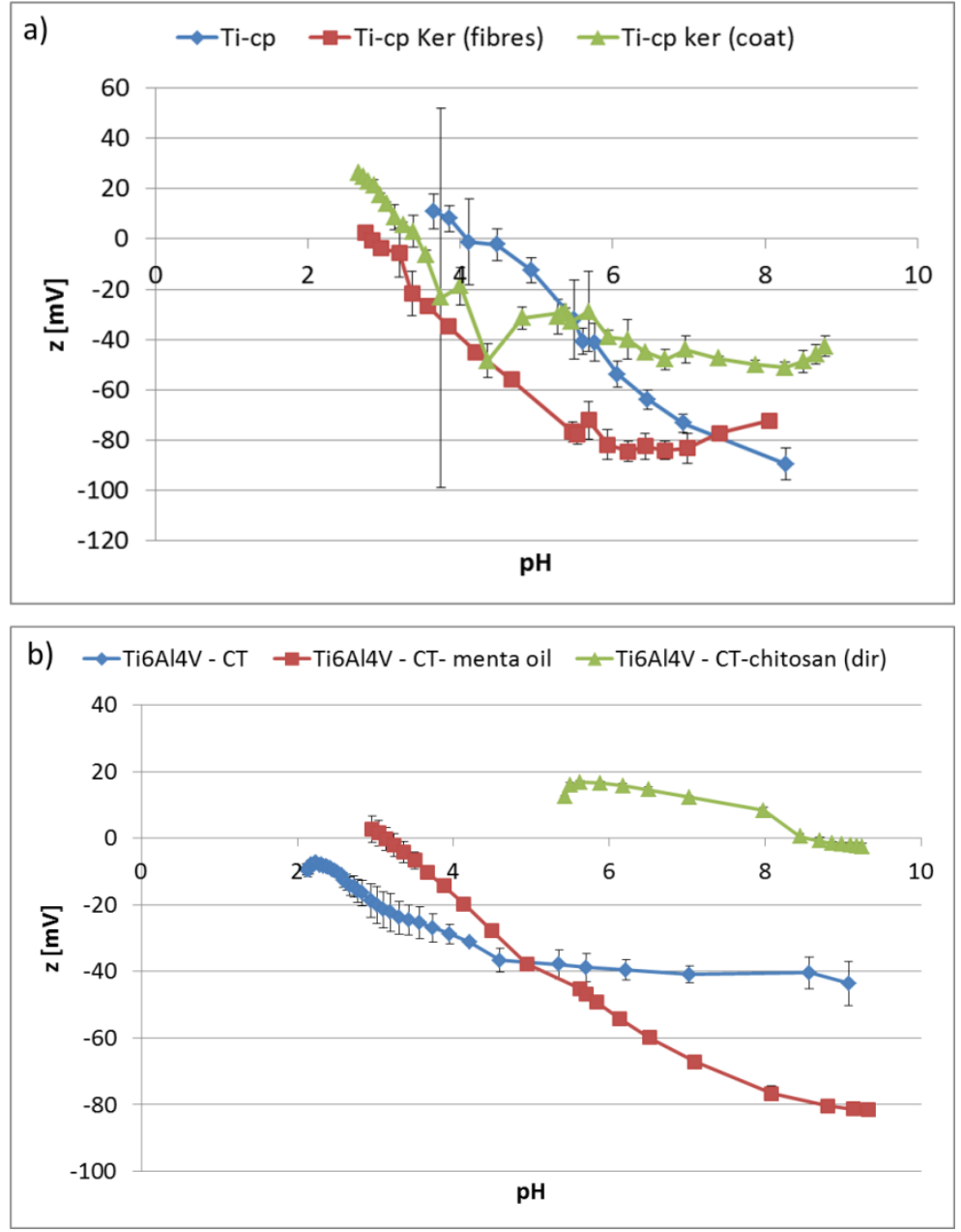

Figure 3. Zeta potential titration curves (a) Ti-cp and its continuous or fibrous coatings, (b) Ti6Al4V$\mathrm{CT}$ and its mint essential oil or chitosan coatings.

Bacterial adhesion (in terms of biofilm formation) was reduced by one order of magnitude on the mint essential oil coatings and by three order of magnitude on the silver doped keratin coatings, evidencing mainly a bacteriostatic effect for mint and an active antibacterial activity for the silver doped keratin fibers.

The keratin coated samples resulted completely biocompatible for human gingival fibroblast cells both with and without silver doping. A significant increase in fibroblast proliferation was observed for the keratin coated samples with respect to the bare substrate [6]. Moreover, the aligned keratin fibers are able to drive fibroblast orientation [7].

\section{Conclusion}

Keratin, chitosan and peppermint essential oil coatings were successfully obtained on titanium surfaces. Keratin was obtained from discarded wool, chitosan from shrimp shells and peppermint essential oil from local production with a sustainable use of resources and promotion of local economies. Numerous techniques (such as SEM-EDS, FTIR, fluorescence microscopy, wettability and 
zeta potential measurements) resulted effective and complementary for the complete chemical and physical characterization of the coatings.

All the coatings have good adhesion to the substrates, good stability in water-based media and the ability to protect the metallic substrate. The keratin coatings are hydrophilic while the mint oil and chitosan coatings are hydrophobic. At the physiological $\mathrm{pH}$, the keratin and mint oil coatings are negatively charged while the chitosan ones are positively charged.

The biological tests evidenced that the oriented keratin fibers are able to drive fibroblast alignment and that the Ag-doped keratin fibers and mint coatings have antibacterial properties.

In conclusion, the here proposed natural coatings, obtained from abundant byproducts or local productions, are promising for the improvement of the biological properties of titanium substrates with a sustainable use of the resources.

Acknowledgments: This activity was partially funded by the European Commission, Project NAT4MORE (NATural molecules on the surface of bioactive materials FOR MOdulating the host REsponse to implants) M.ERANET Call 2016.

\section{References}

1. Yamauchi, K.; Maniwa, M.; Mori, T. Cultivation of fibroblast cells on keratin-coated substrata. J. Biomater. Sci. Polym. Ed. 1998, 9, 259-270, doi:10.1163/156856298x00640.

2. Tachibana, A.; Furuta, Y.; Takeshima, H.; Tanabe, T.; Yamauchi, K. Fabrication of wool keratin sponge scaffolds for long-term cell cultivation. J. Biotechnol. 2002, 93, 165-170, doi:10.1016/s0168-1656(01)00395-9.

3. Cochis, A.; Ferraris, S.; Sorrentino, R.; Azzimonti, B.; Novara, C.; Geobaldo, F.; Giachet, F.T.; Vineis, C.; Varesano, A.; Abdelgeliel, A.S.; et al. Silver-doped keratin nanofibers preserve a titanium surface from biofilm contamination and favor soft-tissue healing. J. Mater. Chem. B 2017, 5, 8366-8377, doi:10.1039/c7tb01965c.

4. Costa, E.M.; Silva, S.; Veiga, M.; Tavaria, F.K.; Pintado, M.M. A review of chitosan's effect on oral biofilms: Perspectives from the tube to the mouth. J. Oral Biosci. 2017, 59, 205-210.

5. Singh, R.; Shushni, M.A.M.; Belkheir, A. Antibacterial and antioxidant activities of Mentha piperita L. Arab. J. Chem. 2015, 8, 322-328, doi:10.1016/j.arabjc.2011.01.019.

6. Ferraris, S.; Giachet, F.T.; Miola, M.; Bertone, E.; Varesano, A.; Vineis, C.; Cochis, A.; Sorrentino, R.; Rimondini, L.; Spriano, S. Nanogrooves and keratin nanofibers on titanium surfaces aimed at driving gingival fibroblasts alignment and proliferation without increasing bacterial adhesion. Mater. Sci. Eng. C 2017, 76, 1-12, doi:10.1016/j.msec.2017.02.152.

7. Ferraris, S.; Guarino, V.; Cochis, A.; Varesano, A.; Maya, I.C.; Vineis, C.; Rimondini, L.; Spriano, S. Aligned keratin submicrometric-fibers for fibroblasts guidance onto nanogrooved titanium surfaces for transmucosal implants. Mater. Lett. 2018, 229, 1-4, doi:10.1016/j.matlet.2018.06.103.

8. Varesano, A.; Vineis, C.; Tonetti, C.; Ramirez, D.O.S.; Mazzuchetti, G. Chemical and physical modifications of electrospun keratin nanofibers induced by heating treatments. J. Appl. Polym. Sci. 2014, 131, 40532, doi:10.1002/app.40532.

9. Ferraris, S.; Spriano, S.; Pan, G.; Venturello, A.; Bianchi, C.L.; Chiesa, R.; Faga, M.G.; Maina, G.; Vernè, E. Surface modification of Ti-6Al-4V alloy for biomineralization and specific biological response: Part I, inorganic modification. J. Mater. Sci. Mater. Electron. 2011, 22, 533-545, doi:10.1007/s10856-011-4246-2.

10. Cazzola, M.; Ferraris, S.; Allizond, V.; Bertea, C.M.; Novara, C.; Cochis, A.; Geobaldo, F.; Bistolfi, A.; Cuffini, A.; Rimondini, L.; et al. Grafting of the peppermint essential oil to a chemically treated Ti6Al4V alloy to counteract the bacterial adhesion. Surf. Coatings Technol. 2019, 378, 125011, doi:10.1016/j.surfcoat.2019.125011.

11. Cazzola, M.; Ferraris, S.; Banche, G.; Di Confiengo, G.G.; Geobaldo, F.; Novara, C.; Spriano, S. Innovative Coatings Based on Peppermint Essential Oil on Titanium and Steel Substrates: Chemical and Mechanical Protection Ability. Mater. 2020, 13, 516, doi:10.3390/ma13030516.

12. Curcio, M.; Blanco-Fernandez, B.; Diaz-Gomez, L.; Concheiro, A.; Alvarez-Lorenzo, C. Hydrophobically Modified Keratin Vesicles for GSH-Responsive Intracellular Drug Release. Bioconjugate Chem. 2015, 26, 1900-1907, doi:10.1021/acs.bioconjchem.5b00289. 
13. Bal, B.S.; Rahaman, M. Orthopedic applications of silicon nitride ceramics. Acta Biomater. 2012, 8, 2889-2898, doi:10.1016/j.actbio.2012.04.031.

14. Ferraris, S.; Yamaguchi, S.; Barbani, N.; Cazzola, M.; Cristallini, C.; Miola, M.; Vernè, E.; Spriano, S. Bioactive materials: In vitro investigation of different mechanisms of hydroxyapatite precipitation. Acta Biomater. 2020, 102, 468-480, doi:10.1016/j.actbio.2019.11.024.

15. Ferraris, S.; Cazzola, M.; Peretti, V.; Stella, B.; Spriano, S. Zeta Potential Measurements on Solid Surfaces for in Vitro Biomaterials Testing: Surface Charge, Reactivity Upon Contact With Fluids and Protein Absorption. Front. Bioeng. Biotechnol. 2018, 6, 60, doi:10.3389/fbioe.2018.00060.

16. Swain, S.K.; Dey, R.K.; Islam, M.; Patel, R.K.; Jha, U.; Patnaik, T.; Airoldi, C. Removal of Fluoride from Aqueous Solution Using Aluminum-Impregnated Chitosan Biopolymer. Sep. Sci. Technol. 2009, 44, 20962116, doi:10.1080/01496390902881212.

(C) 2020 by the authors. Submitted for possible open access publication under the terms and conditions of the Creative Commons Attribution (CC BY) license (http://creativecommons.org/licenses/by/4.0/). 